\title{
A 16-year-old boy with progressive central vision loss in his left eye
}

previously well 16-year-old Aboy presented to the emergency department with a 1month history of a painless, progressive loss of central vision in his left eye. He was otherwise asymptomatic and had no history of fever, infection, recent vaccination, toxic exposure or trauma. He had no diploplia, seizures, bowel or bladder dysfunction, or changes in sensory or motor function or in level of consciousness. His colour vision was unaffected.

On physical examination, the patient appeared well, was afebrile - and had normal vital signs. His visual acuity was normal for his right eye but was reduced in his left eye. Except for deep-tendon hyper-reflexia in his left lower limb, results of his neurological examination, including gross and fine sensation, gait, power, motor
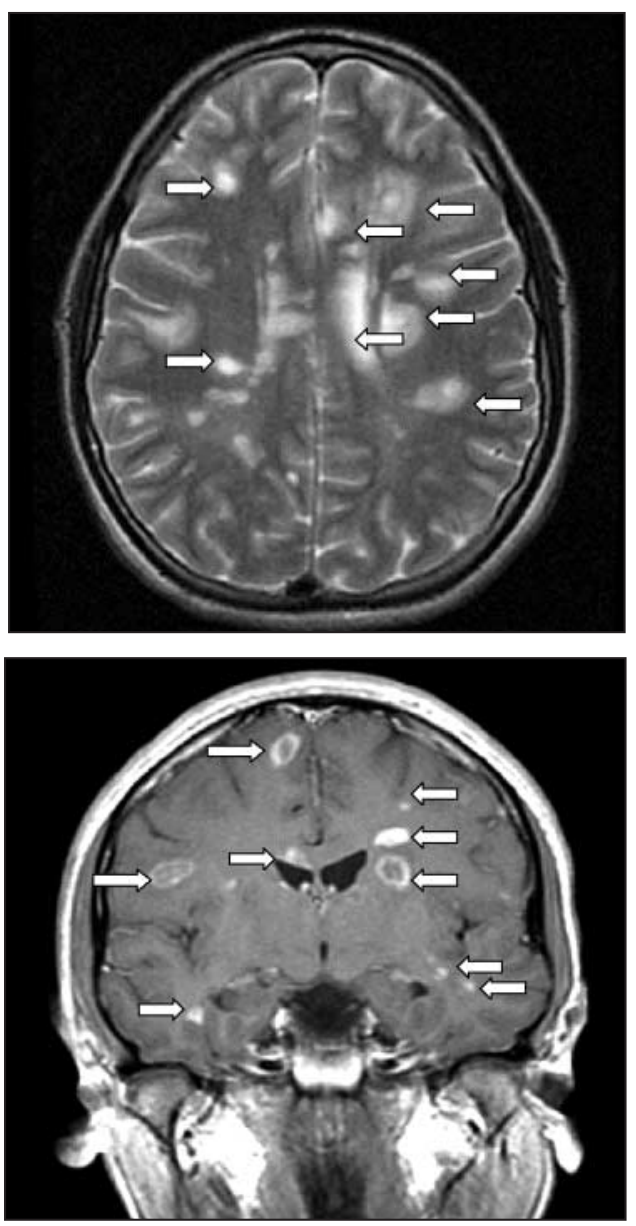

and cerebellar tests, were normal. Results of cranial nerve and funduscopic examinations were also normal, but he had bilateral gaze-evoked horizontal and vertical nystagmus. A lumbar puncture produced clear cerebrospinal fluid (CSF) with normal cell counts and glucose, protein and lactate levels, and no oligoclonal banding. Full work-up of the CSF for viral and bacterial encephalitis was negative. Other laboratory tests were all normal.

A head CT scan was consistent with demyelination. Brain MRI (Figs. 1 and 2) showed multiple supratentorial lesions within the periventricular, deep and subcortical white matter of the frontal temporal, parietal and occipital lobes and corpus callosum (arrows) as well as several infratentorial lesions within the pons, medulla, and cerebellar hemispheres.

Multiple sclerosis (MS) was considered a likely diagnosis, but acute disseminated encephalomyelitis (ADEM) could not be ruled out, so the patient was treated with a course of high-dose intravenous therapy with methylprednisolone for 5 days. His visual acuity improved but did not return to baseline. He was discharged home on a tapering dose of oral steroid therapy, with ongoing neurological follow-up.

MS is a slowly progressive disease characterized by exacerbations and remissions. It usually develops between the ages of 20 and 40 years but can first present in childhood. ${ }^{1}$ MS is diagnosed using both clinical features of the disease and tests such as visual-evoked potentials and MRI. ${ }^{2}$ Signs and symptoms need to demonstrate both dissemination in time and space for a diagnosis of MS.

ADEM is a monophasic inflammatory condition that resembles MS but that typically occurs in younger adults or children. Patients often present with ataxia, pyramidal motor signs, disturbed consciousness, headache and cranial nerve abnormalities. ADEM often occurs following a viral infection or, more rarely, vaccination. Although the mortality was once felt to be as high as $20 \%$, recent case series suggest death is rare, an improvement possibly due to the nearly routine use of systemic steroids. In general, ADEM is self-limited and rarely leads to long-term sequelae, although relapses are possible.

MRI usually enables differentiation between MS and ADEM. On MRI, MS patients usually have periventricular white matter and corpus callosum changes but no involvement of the deep grey matter. In contrast, less than $30 \%$ of patients with ADEM will have lesions in these locations, but $40 \%$ will have thalamic involvement, which is exceedingly rare in MS.

Although our patient's clinical and radiologic presentation was more characteristic of MS than of ADEM, only optic neuritis, and not MS, could be diagnosed in the absence of new lesions or a second clinical attack.

\section{Shaun Morris \\ Linda Hiraki \\ Aleixo Muise \\ Department of Pediatrics \\ Hospital for Sick Children \\ Toronto, Ont.}

\section{References}

1. Ghezzi A, Deplano V, Faroni J, Grasso MG, Liguori M, Marrosu G, et al. Multiple sclerosis in childhood: clinical features of 149 cases. Mult Scler 1997; 3(1):43-6.

2. McDonald WI, Compston A, Edan G, Goodkin D, Hartung HP, Lublin FD, et al. Recommended diagnostic criteria for multiple sclerosis: guidelines from the International Panel on the Diagnosis of Multiple Sclerosis. Ann Neurol 2001;50(1):121-7.

3. Hynson JL, Kornberg AJ, Coleman LT, Shield L, Harvey AS, Kean MJ. Clinical and neuroradiologic features of acute disseminated encephalomyelitis in children. Neurology 2001;56(10);1308-12. 